ROTHE, E. H.

Math. Zeitschr. Bd. 63, S. 195-218 (1955)

\title{
Mapping degree in Banach spaces and spectral theory.
}

\author{
To the memory of I. SCHUR in gratitude.
}

\author{
By
}

\section{ERICH H. ROTHE.}

1. Introduction. The mapping degree in a real Banach space $R$ was established by Leray and Schauder ${ }^{1}$ ) for mappings of a bounded domain $D$ of $R$ into $R$ which are of the form

$$
y=f(x)=x-F(x)
$$

where $F(x)$ is completely continuous. This was done by assuming Brouwer's theory of the mapping degree in finite dimensional Euclidean spaces as known, and then using an approximation procedure based on the fact that to every $\varepsilon>0$ there exists a map $S(x)$ of $D$ into a finite dimensional subspace of $R$ such that

$$
\|F(x)-S(x)\|<\varepsilon .
$$

In the present paper an attempt is made to develop the degree theory directly in the Banach space $R$. Here already the case that (1.1) is a linear map requires some consideration due to the fact that no orientation is defined in a Banach space $\left.{ }^{2}\right)$. Accordingly, the main part of this paper deals with the linear case. The problem is to assign to a linear non-singular map (1.1) an index $j=j(f)$ which is +1 or -1 , is invariant under homotopy, and +1 if $f$ is the identity map $I$. It is natural to consider $x-\mu F(x)$ for $0 \leq \mu \leq 1$, or with $\lambda=\mu^{-1}$

$$
f_{\lambda}(x)=(\lambda I-F)(x)
$$

and the spectrum $\sigma(F)$ of $F$. If the part of $\sigma(F)$ with $\Re(\lambda) \geq 1^{3}$ ) contains only a finite number of points and if it is possible to assign to each such point

1) [6]. The theory was extended to linear convex topological spaces by Leray [5], and Nagumo [9]. (Numbers in brackets refer to the bibliography at the end of the paper.) The present paper is restricted to Banach spaces. As usual the norm of an element $x$ of such a space will be denoted by $\|x\|$.

2) In a finite dimensional space the case of a linear map (1.1) is quite trivial, the degree being +1 or -1 according to whether the map (1.1) preserves the orientation or not, i.e., whether the determinant of $(1.1)$ is $>0$ or $<0$. However, it seems to be an unsolved problem whether in case of a linear completely continuous Banach space map $F(x)$ the approximation (1.2) can be effected by a linear $S$. Therefore in order to establish the degree theory for linear maps (1.1) in the Banach space by the approximation method one would (at least at the present state of our knowledge) have to use the degree theory for non linear maps in the finite dimensional space.

3) $\Re(\lambda)$ denotes the real part of the complex number $\lambda$. 
a positive integer $m_{i}$ such that

$$
j=\sum_{i}(-1)^{m i}
$$

is invariant under homotopy ${ }^{4}$ ), then (1.4) will represent a suitable definition for the index $j^{5}$ ). This program is carried through in section 2. In this setup the homotopy theorem becomes a theorem on spectral perturbation, and, in order to use fully the spectral perturbation thepry, we deal in that section with linear maps of a complex Banach space $X$ into itself. In section 3 the special situation is treated where $X$ admits a "conjugation" and where, consequently, it is possible to define "real". maps. Certain simplification in the basic assumptions of section 2 and in the definition (2.4) of the index can be made in this case. Section 4 deals with homotopy properties of real linear maps of such a space $X$ into itself. Section 5 treats the non linear case in an arbitrary, complex Banach space $X$, but only "smooth" mappings (def. 5.1) are considered while the treatment of mappings with less restrictive assumptions is left to further investigation. The results of section 5 for complex spaces are in section 6 carried over to real Banach spaces $R$ by imbedding them (def. 6.1) in a complex Banach space.

2. The indeX of a IINEAR Map in a Complex Banach space $X$. Let $X$ be a complex Banach space and $F$ a bounded linear map of $X$ into itself which satisfies the following assumptions $A, B$, and $C$ :

Assumption A. No point of $\Re(\lambda)=1$ belongs to the spectrum $\sigma(F)$ of $F$.

Assumption B. The intersection of $\sigma(F)$ with $\Re(\lambda)>1$ consists of isolated points.

Before formulating Assumption $C$ we remark first that $B$ together with the boundedness of $F$ implies that the intersection $\sigma(F) \cap\{\mathfrak{\Re}(\lambda)>1\}$ consists of a finite number of points $\lambda_{1}, \lambda_{2}, \ldots, \lambda_{r}$, or is empty. Then, for $i=1,2, \ldots, r$, let $c_{i}$ be the circumference of a counter-clockwise oriented circle with center $\lambda_{i}$ and such that $\dot{\lambda}_{i}$ is the only point of $\sigma(F)$ inside or on $c_{i}$. Let $E_{\lambda_{i}}$ be the projection ${ }^{6}$ ) defined by

$$
E_{\lambda_{i}}=\frac{1}{2 \pi \sqrt{-1}} \int_{c_{i}} R_{\mu}(F) d \mu
$$

where

$$
R_{\mu}(F)=(\mu I-F)^{-1}
$$

Finally; let

$$
X_{\lambda_{i}}=E_{\lambda_{i}} X
$$

be the range of $E_{\lambda_{i}}$.

4) It will be seen that these two properties are certainly true if $F$ is completely continuous.

5) For the Leray-Schauder index the validity of (1.4) is a theorem (cf. [6] p. 58)

while it will be a definition in the present paper.

6) [1], p. 196. 
Assumption C. The dimension of $X_{\lambda_{i}}$ is a finite number $m_{i}{ }^{7}$ ).

Definition 2.1. The index $j=j(f)$ of the mapping (1.1) is defined by

$$
j=j(f)=(-1)^{\sum_{i=1}^{r} m_{i}}
$$

The object of the present section is the proof of the following two theorems:

THeOREM 2.1. Let $F_{0}(x)$ satisfy the assumptions $\mathrm{A}, \mathrm{B}$, and $\mathrm{C}$ and let

$$
f_{0}(x)=x-F_{0}(x) .
$$

Then there exists an $\varepsilon>0$ of the following property: if the linear bounded map (1.1) satisfies

$$
\left\|f-f_{0}\right\|=\left\|F-F_{\mathrm{o}}\right\|<\varepsilon
$$

then $F$ satisfies the same assumptions as $F_{0}$ and

$$
j(f)=j\left(f_{0}\right) \text {. }
$$

THEOREM 2.2. For $0 \leq t \leq 1$ let $f_{t}(x)=x-F_{t}(x)$ where $F_{t}(x)$ satisfies assumptions $\mathrm{A}, \mathrm{B}$, and $\mathrm{C}$, and where in addition $F_{t}$ is continuous in $\left.t^{8}\right)$. Then $j\left(f_{1}\right)=j\left(f_{0}\right)$.

Theorem 2.2 is an immediate consequence of theorem 2.1 as application of the Heine-Borel theorem to the interval $0 \leq t \leq 1$ shows. The proof of theorem 2.1 is based on the following lemmas 2.1 and 2.2 .

LEMMA 2.1. Let $F_{0}$ satisfy the assumptions of theorem 2.1. We denote by $\sigma^{+}\left(F_{0}\right)$ and $\sigma^{-}\left(F_{0}\right)$ those parts of the spectrum $\sigma\left(F_{0}\right)$ of $F_{0}$ which lie in $\Re(\lambda)>1$ and $\Re(\lambda)<1$ respectively such that (by assumption $\mathrm{A}) \sigma\left(F_{0}\right)=\sigma^{+}\left(F_{0}\right)+\sigma^{-}\left(F_{0}\right)$. Let $0^{-}$be an open set in $\Re(\lambda)<1$ containing $\left.\sigma^{-}\left(F_{0}\right)^{9}\right)$, and let, for $i=1,2, \ldots, r_{\mathrm{G}}$, $c_{i}$ be counter-clockwise oriented circles defined as follows: by assumption $\mathrm{B}$, $\sigma^{+}\left(F_{0}\right)$ consists of a finite number of points $\lambda_{1}^{0}, \lambda_{2}^{0}, \ldots, \lambda_{r}^{0} . c_{i}$ is then a circle with center $\lambda_{i}^{0}$ which lies in $\Re(\lambda)>1$ and such that the closed disk bounded by $c_{i}$ contains no other point of $\sigma\left(F_{0}\right)$. Then there exists. a positive $\varepsilon_{0}$ such that for

$$
\left\|F-F_{0}\right\|<\varepsilon_{0}
$$

the spectrum $\sigma(F)$ of $F$ is contained in the open set 0 which is the union of $0^{-}$ and the open disks bounded by the $c_{i}$.

Proor. Since 0 is an open set containing $\sigma\left(F_{0}\right)$ the lemma follows from the well-known fact that the spectrum $\sigma(F)$ of $F$ depends continuously on $F^{10}$ ).

LEMma 2.2. If $F_{0}$ is bounded linear and $\lambda_{i}$ an isolated point of $\sigma\left(F_{0}\right)$ then there exists a positive $\varepsilon_{i}$ such that for all linear bounded $F$ satisfying

$$
\left\|F-F_{0}\right\|<\varepsilon_{i}
$$

7) Assumptions A, B, and C are automatically satisfied if $F(x)$ is completely con-. tinuous. See, e.g., $[1]$ p. 207.

8) I.e., for each $t_{0}$ in $0 \leqq t \leqq 1$ there exists to each $\eta>0$ a $\zeta$ such that $\left\|F_{t}-F_{t_{0}}\right\|<\eta$ or what is the same $\left\|F_{t}(x)-F_{t_{0}}(x)\right\|<\|x\| \eta$ for $\left|t-t_{0}\right|<\zeta$.

$\left.{ }^{9}\right)$ Such set $0^{-}$exists since $\sigma^{-}\left(F_{0}\right)$ is closed.

10) For definition and proof of the continuity of $\sigma(F)$, see $[4]$ p. 118.

Mathematische Zeitschrift. Bd.63. 
the following is true: if $m_{i}(F)$ denotes the dimension of the range of the projection

$$
\frac{1}{2 \pi \sqrt{-1}} \int_{c_{i}} R_{\mu}(F) d \mu
$$

and if $m_{i}\left(F_{0}\right)$ is finite then $m_{i}(F)$ is finite and

$$
m_{i}(F)=m_{i}\left(F_{0}\right) \text {. }
$$

We postpone the proof and show first that lemmas 2.1 and 2.2 together imply theorem 1. Using the notations used in the formulation of the two lemmas, we choose

$$
\varepsilon=\min \left(\varepsilon_{0}, \varepsilon_{1}, \varepsilon_{2}, \ldots, \varepsilon_{r}\right)
$$

and will show that with this choice of $\varepsilon$, theorem 2.1 is true. That $F$ satisfies assumption $A$ is obvious from lemma 2.1. To prove assumption $B$ we note first that lemma 2:1 also, implies that the part of $\sigma(F)$ with $\Re(\lambda)>1$ is contagined in the open disks $o_{i}$ bounded by the circles $c_{i}$. Since there are only a finite number of these disks it will be sufficient to prove that for fixed $i$. the intersection $\sigma_{i}=o_{i} \cap \sigma(F)$ is finite. Now by the assumption made on $F_{0}$, $m_{i}\left(F_{0}\right)$ is finite (Assumption C). Therefore, by lemma 2.2, $m_{i}(F)$ is also finite; i.e., the range $X_{i}$ of the projection (2.10) is a finite dimensional space. Now $\sigma_{i}$ is a spectral set of $\left.F^{11}\right)$. Therefore, by a theorem of Dunford $\left.{ }^{12}\right) \sigma_{i}$ is identical with the spectrum of the restriction $F_{\mid X_{i}}$ of $F$ to the finite dimensional space $X_{i}$ and therefore certainly finite. Thus assumption B is proved, and assumption $C$ follows now easily: denote by $\lambda_{1}^{i}, \lambda_{2}^{i}, \ldots, \lambda_{i_{n}}^{i}$ the points of the set $\sigma_{i}$ which just has been proved to be finite. Let $o_{j}^{i}$ be disjoint circular disks with centers $\lambda_{j}^{i}$ and lying in $o_{i}$. Let $c_{j}^{i}$ be the counter-clockwise oriented boundary of $o_{j}^{i}$. Then

$$
\frac{1}{2 \pi V-1} \int_{c_{i}} R_{\mu}(F) d \mu=\sum_{j=1}^{i_{n}} \frac{1}{2 \pi V-1} \int_{c_{j}^{i}} R_{\mu}(F) d \mu .
$$

Therefore, $X_{i}$ is the direct sum of the ranges $X_{j}^{i}$ of the projections which are the terms of the right hand sum in (2.13) and

$$
m_{i}(F)=\sum_{j=1}^{i_{n}} m_{j}^{i}(F)
$$

if $m_{j}^{i}(F)$ denotes the dimension of $X_{j}^{i}$. Since $m_{i}(F)$ has alrea ly been proved to be finite, (2.14) implies that each $w_{i}^{i}(F)$ is finite which is assumption $C$. At the same time (2.7) follows now from (2.14), (2.11), and definition 2.1.

It remains to prove lemma 2.2. This lemma will be a consequence of four further lemmas.

$$
\text { p. } 196 .
$$

11) A spectral set of $F$ is a subset of $\sigma(F)$ which is open and closed in $\sigma(F)$. Cf. [l] 
LEMMA 2.3. Let $B_{0}$ be a linear bounded operator on $X$ whose bounded inverse $B_{0}^{-1}$ exists. Let $B$ be a bounded linear operator which satisfies the inequality

$$
\left\|B-B_{0}\right\|<\frac{1}{2}\left\|\dot{B}_{0}^{-1}\right\|^{-1}
$$

Then $B^{-1}$ exists and satisfies the inequality

$$
\left\|B^{-1}-B_{0}^{-1}\right\|<2\left\|B_{0}^{-1}\right\|^{2}\left\|B-B_{0}\right\| .
$$

Proof. The fact that $(2,15)$ implies the existence of $B^{-1}$ and the inequality

$$
\left\|B^{-1}-B_{0}^{-1}\right\|<\frac{\left\|B_{0}^{-1}\right\|^{2}\left\|B-B_{0}\right\|}{1-\left\|B-B_{0}\right\|\left\|B_{0}^{-1}\right\|}
$$

is well known ${ }^{13}$ ): But (2.17) together with (2.15) obviously implies (2.16).

LEMMA 2.4. Let $A_{0}$ be a linear bounded operator on $X$. Let $C$ be a closed bounded subset of the resolvent set $\varrho\left(A_{0}\right)$ of $A_{0}$. Then the resolvent $R_{\lambda}(A)$ of $A$ is continuous at $A=A_{0}$ for $\lambda C C$, and the continuity is uniform as $\lambda$ varies over $C$.

Proof. As is well known for each $x<X, R_{\lambda}\left(A_{0}\right)(x)$, and therefore also $\left\|R_{\lambda}\left(A_{0}\right)(x)\right\|$, is continuous for $\lambda<\varrho\left(A_{0}\right)$. Consequently, the latter real valued function of $\lambda$ reaches a maximum in the closed bounded set $C$ and we have for suițable positive $M_{x}$ the inequality

$$
\left\|R_{\lambda}\left(A_{0}\right)(x)\right\|<M_{x} \text { for } \lambda \text { in } C \text { and all } x<X .
$$

By a well-known theorem ${ }^{14}$ ) (2.16) implies the existence of a constant $M$ such that

$$
\left\|R_{\lambda}\left(A_{0}\right)\right\|<M \quad \text { for } \lambda<C .
$$

Let now $A$ be such that

$$
\left\|A-A_{0}\right\|<\frac{1}{2 M} .
$$

We have then by $(2.18)$ and (2.17)

$$
\left\|\left(\lambda I-A_{0}\right)-(\lambda I-A)\right\|=\left\|A-A_{0}\right\|<\frac{1}{2 M}<\frac{1}{2\left\|R_{\lambda}\left(A_{0}\right)\right\|}=\frac{1}{2\left\|\left(\lambda I-A_{0}\right)^{-1}\right\| !} .
$$

From this inequality we conclude by applying lemma 2.3 with $B_{0}=\lambda I-A$ $B=\lambda I-A$ that $R_{\lambda}(A)=B^{-1}$ exists and satisfies the inequality

$$
\left\|R_{\lambda}(A)-R_{\lambda}\left(A_{0}\right)\right\|<2\left\|R_{\lambda}\left(A_{0}\right)\right\|^{2}\left\|B-B_{0}\right\|=2\left\|R_{\lambda}\left(A_{0}\right)\right\|^{2}\left\|A-A_{0}\right\|
$$

which because of $(2.17)$ proves the lemma.

Lemma 2.5. Let $c$ be a rectifiable curve in the resolvent set of $A_{0}$. Then

is continuous at $A=A_{0}$.

$$
\int_{c} R_{\mu}(A) d \mu
$$

\footnotetext{
13) $[4]$ p. 92 .

14) See, e.g., [4] p. 26, Theorem 2.12.2.
} 
Proof. The lemma is an immediate consequence of the preceding lemma.

LEMMA $2.6^{15}$ ). Let $E_{0}$ be a projection whose range has the finite dimension $m_{0}$, and let $E$ be a projection for which

$$
\left\|E-E_{0}\right\|<\frac{1}{2\left\|E_{0}\right\|} .
$$

Then the range of $E$ has a finite dimension $m$ and $m=m_{0}$.

PROOF OF LEMMA 2.2. Let $E_{0}$ be the projection obtained from (2.10) by replacing $F$ by $F_{0}$. By lemma 2.5 we can choose an $\varepsilon_{i}$ such that for $F$ satisfying (2.9) the inequality (2.19) holds with $E$ being the projection (2.10), and the equality $(2.11)$ to be proved follows now from lemma 2.6 .

3. REAL TRANSFORMATIONS IN A COMPLEX BANACH SPACE. In this section we consider "real" transformations in certain complex Banach spaces (definition 3.3 ${ }^{16}$ ). It turns out that for such țransformations in the definition (2.4) of the index only those $m_{i}$ have to be considered which correspond to real points of the spectrum (Theorem 3.1). In addition, some simplifications in assumpttons $\mathrm{A}, \mathrm{B}$, and $\mathrm{C}$ of section 2 are possible (Theorem 3.2).

Definition 3.1. Let $X$ be a complex Banach space with elements $x, y, \ldots$ A map $x \rightarrow \bar{x}$ of $X$ onto itself is called a conjugation if it satisfies the following four properties:

$$
\begin{aligned}
\overline{\bar{x}} & =x \\
\bar{x}+\bar{y} & =\bar{x}+\bar{y} \\
\|x\| & =\|\bar{x}\| \\
\overline{\lambda x} & =\bar{\lambda} \bar{x}
\end{aligned}
$$

where $\bar{\lambda}$ is the conjugate complex number to the complex number $\lambda$.

Definition 3.2. Let $x \rightarrow \bar{x}$ be a conjugation in $X$. The elements $x$ for which $x=\bar{x}$ are called real (with respect to the given conjugation).

Lemma 3.1. Let $x \rightarrow \bar{x}$ be a conjugation in $X$. $^{-}$Then the real elements of $X$ (with their original norms) form a Banach space $R$ over the reals.

The proof consists in a rather obvious verification. We omit the details.

LEMMA 3.2. Let $x \rightarrow \bar{x}$ be a conjugation in $X$, and let $R$ be the real Banach space of the preceding lemma. Then every element $x$ of $X$ can be witten uniquely as (3.5)

$$
x=x^{\prime}+i x^{\prime \prime}
$$

where $x^{\prime}$ and $x^{\prime \prime}$ are elements of $R$ and $i=\sqrt{-1}$.

Proof. It is easily seen that $x^{\prime}=(x+\bar{x}) / 2, x^{\prime \prime}=(x-\bar{x}) / 2 i$ is the unique couple satisfying the requirements.

- Definition 3.3. Let $X$ and $R$ have the same meaning as in lemma 3.1. Then a continuous linear map $A$ of $X$ into itself or into another space with a conjugation is called real if

$$
A(\bar{x})=\overline{A(x)}
$$

15) For this lemma and its proof, see $[10]$ p. 424 . Lemma 4.

16) Cf. the similar definitions for the Hilbert space case given in [11], chapter IX, $\$ 2$. 
Lemma 3.3. The continuous linear map $A$ of $X$ into itself is real if and only if it leaves $R$ invariant.

We omit the obvious proof.

Lemma 3.4. If $A$ is real then (i) the spectrum $\sigma(A)$ of $A$ is symmetric with respect to the real axis of the complex $\lambda$-plane and (ii)

$$
R_{\bar{\lambda}}(A)(x)=\overline{R_{\lambda}(A)(\bar{x})}
$$

Proof. Since $\sigma(A)$ and the resolvent set $\varrho(A)$ of $A$ are disjoint sets whose union is the whole $\lambda$-plane it will for the proof of i) be sufficient to show that $\varrho(A)$ is symmetric with respect to the real axis. Let then $\lambda_{0}<\varrho(A)$; we have to prove that $\bar{\lambda}_{0}<\varrho(A)$. By definition of the resolvent we have

$$
\left(\lambda_{0} I-A\right) R_{\lambda_{0}}(A)(x)=x \text { for all } x<X .
$$

Since $A$ is real it follows from lemma 3.3 that for all $y<X$

$$
\left(\bar{\lambda}_{0} I-A\right)(\bar{y})=\overline{\left(\lambda_{0} I-A\right)(y)}
$$

and therefore from (3.8)

$$
\left(\vec{\lambda}_{0} I-A\right) \overline{R_{\lambda_{0}}(A)(x)}=\overline{\left(\lambda_{0} I-A\right) \overline{R_{\lambda_{0}}(A)(x)}}=\bar{x},
$$

or replacing $x$ by $\bar{x}$

$$
\left(\bar{\lambda}_{0} I-A\right) \cdot \overline{R_{\lambda_{g}}(A)(\bar{x})}=x
$$

We set now

$$
S(x)=\overline{R_{\lambda_{0}}(A)(\bar{x})}
$$

$S(x)$ is bounded and as (3.9) shows a right inverse of $\bar{\lambda}_{0} I-A$. Interchanging the two factors of the left member of $(3.8)$ we would have seen that $S(x)$ is also a bounded left inverse. Thus $\bar{\lambda}_{0} I-A$ has a uniquely determined bounded inverse which shows that $\bar{\lambda}_{0}$ is in $\varrho(A)$ and thus proves i). Moreover, since $S(x)$ is the bounded inverse of $\bar{\lambda}_{0} I-A$ we see from the definition of the resolvent that $S(x)=R_{\bar{\lambda}_{0}}(A)(x)$. Comparison with (3.10) proves (3.7).

LEMMA 3.5. Let $A$ be real and $\lambda_{0}$ an isolated point of the spectrum $\sigma(A)$ of $A$ such that by the preceding lemma $\bar{\lambda}_{0}$ is also an isolated point of $\sigma(A)$. Let $c_{0}$ be a counter-clockwise oriented circle with center $\lambda_{0}$ and such that the closed disk bounded by $c_{0}$ contains no other point of $\sigma(A)$ but $\lambda_{0}$, and let $c_{0}^{\prime}$ be the counterclockwise oriented circle with center $\bar{\lambda}_{0}$ and of the same radius as $c_{0}$. Finally; let $X_{\lambda_{0}}, X_{\bar{\lambda}_{0}}$ denote the ranges of the projections

$$
E_{\lambda_{0}}=\frac{1}{2 \pi \sqrt{-1}} \int_{c_{0}} R_{\mu}(A) d \mu, \quad E_{\bar{\lambda}_{0}}=\frac{1}{2 \pi \sqrt{-1}} \int_{c_{0}^{\prime}} R_{\mu}(A) d \mu
$$

respectively. We then claim:

i) if $\lambda_{0}$ is not real then $X_{\lambda_{0}}$ and $X_{\bar{\lambda}_{0}}$ are conjugate to each other (i.e., if $y$ varies over $X_{\lambda_{0}}$ then $\bar{y}$ varies over $\left.X_{\bar{\lambda}_{0}}\right)$, and, consequently, they are of the same dimension;

ii) if $\lambda_{0}$ is real then $X_{\lambda_{0}}$ is invariant under conjugation. 
Proof. Since $\bar{x}$ varies over all of $X$ as $x$ does, $X_{\lambda_{0}}$ may be characterized as the set of all $E_{\dot{\lambda}_{0}}(\bar{x})$ as $x$ varies over $X$, and since $X_{\bar{\lambda}_{0}}$ is the set of all $E_{\bar{\lambda}_{0}}(x)$ as $x$ varies over $X$, it is clear that for the proof of i) it is sufficient to prove

$$
E_{\bar{\lambda}_{0}}(x)=\overline{E_{\lambda_{0}}(\bar{x})} \text {. }
$$

But (3.12) implies also ii), for in the case of a real $\lambda_{0}$ it reads $\overline{E_{\lambda_{0}}(\bar{x})}=E_{\lambda_{0}}(x)$. Thus it remains to prove $(3.12)$ : let $r$ be the common radius of $c_{0}$ and $c_{0}^{\prime}$. We set $\mu=\bar{\lambda}_{0}+r e^{i \varphi}, \lambda=\lambda_{0}+r e^{-i \varphi}$ such that $\mu=\bar{\lambda}$. We then have from (3.11) and (3.7)

$$
\begin{aligned}
& E_{\bar{\lambda}_{0}}(x)=\frac{1}{2 \pi} \int_{0}^{2 \pi} R_{\mu}(A)(x) r e^{i \varphi} d \varphi=\frac{1}{2 \pi} \int_{0}^{2 \pi} R_{\bar{\lambda}}(A)(x) r e^{i \varphi} d \varphi \\
& =\frac{1}{2 \pi} \int_{0}^{2 \pi} \overline{R_{\lambda}(A)(\bar{x}) r e^{-i \varphi} d \not} \\
& =-\frac{1}{2 \pi i} \int_{0}^{2 \pi} R_{\lambda}(A)(\bar{x})\left(-i r e^{-i \varphi} d \varphi\right) \\
& =\overline{-\frac{1}{2 \pi i} \int_{-c_{0}} R_{\lambda}(A)(\bar{x}) d \lambda}=\overline{\frac{1}{2 \pi i} \int_{c_{0}} R_{\lambda}(A)(\bar{x}) d \lambda}=\overline{E_{\lambda_{0}}(\bar{x}) .}
\end{aligned}
$$

LemMA 3.6. Let. $F$ satisfy the assumptions $\mathrm{A}, \mathrm{B}$, and $\mathrm{C}$ of section 2 and, in addition, be real. Let $\epsilon$ be a simply closed rectitiable curve which is oriented in the counter-clockwise sense, lies in the half plane $\Re(\lambda)>1$, and encloses in its. interior the part of $\sigma(F)$ which lies in $\Re(\lambda)>1$. Then the range of the projection

$$
E=\frac{1}{2 \pi i} \int_{C} R_{\mu}(F) d \mu
$$

is of finite dimension and invariant under conjugation.

Proof. Let $\sigma_{C}$ be the part of the spectrum $\sigma(F)$ which is enclosed by $C$. $\sigma_{C}$ consists of a finite number of points. Now $\mu<\sigma_{C}$ implies $\bar{\mu}<\sigma_{C}$ by lemma 3.4. Therefore the points of $\sigma_{C}$ can be written as $\mu_{1}, \bar{\mu}_{1}, \ldots, \mu_{r}, \bar{\mu}_{r}, \lambda_{1}, \ldots, \lambda_{p}$ where the $\mu$ 's are the different non real points of $\sigma_{C}$ and the $\lambda$ 's the different real points of $\sigma_{C}$. If then the projections $E_{\mu_{j}}, E_{\bar{\mu}_{j}}, E_{\lambda_{j}}$ are defined as in (2.1) we have

$$
E=\sum_{j=1}^{r}\left(E_{\mu_{j}}+E_{\bar{\mu}_{j}}\right)+\sum_{j=1}^{p} E_{\lambda_{j}}
$$

It now follows from assumption $C$ (section 2) that the range of $E$ is of finite dimension and the invariance of this range is [because of (3.14)] a consequence of lemma 3.5 .

THEOREM 3.1. Let $f=I-F$ where $F$ satisfies the assumptions of lemma 3.6. Let $\lambda_{1}, \ldots, \lambda_{p}$ be the real eigenvalues $>1$ of $F$. Let $E_{\lambda_{i}}$ be the projection (2.1), and $m_{i}$ the dimension of its range. Then the index $j(f)$ of $f$ is given by

$$
j(f)=(-1) \sum_{i=1}^{p} m_{i}
$$


Proof: As in the proof of lemma 3.6 let $\mu_{1}, \bar{\mu}_{1}, \ldots, \mu_{r}, \bar{\mu}_{r}$ be the distinct non real eigenvalues of $F$ with real part $>1$, and $E_{\mu_{i}}$, $E_{\vec{\mu}_{i}}$, the corresponding projections. Let $m_{i}^{\prime}, m_{i}^{\prime \prime}$ be the dimensions of the ranges of these projections. Then by definition (2.4)

$$
j(f)=(-1)^{s}
$$

where

$$
s=\sum_{i=1}^{r}\left(m_{i}^{\prime}+m_{i}^{\prime \prime}\right)+\sum_{i=1}^{p} m_{i} .
$$

But by lemma 3.5, $m_{i}^{\prime}=m_{i}^{\prime \prime}$. Therefore

and (3.15) follows from (3.16).

$$
s \equiv \sum_{i=1}^{p} m_{i} \cdot \bmod 2
$$

We will now show that for real transformations the set of assumptions $A$, $B, C$ may be replaced by the following less restrictive set of assumptions:

Assumption $A^{\prime}$. (1.1) is not singular [i.e., $\lambda=1$ does not belong to $\sigma(F)$ :

Assumption $\mathrm{B}^{\prime} . \sigma(F)$ is discrete for $\Re(\lambda) \geq 1$.

Assumption $\mathrm{C}^{\prime}$ is the same as $\mathrm{C}$ except that the eigenvalues in question may have a real part $=1^{17}$ ).

THEOREM 3.2. Theorems 3.1, 2.1, and 2.2 are still true if assumptions $\mathrm{A}$, $\mathrm{B}, \mathrm{C}$ are replaced by $\mathrm{A}^{\prime}, \mathrm{B}^{\prime}, \mathrm{C}^{\prime}$ provided $F$ is real.

Pkoor: Inspection of the proof of theorem 3.1 and the lemmas leading up to it shows that these proofs with obvious modifications still hold under the new assumptions if the curve $C$ of lemma 3.6 is replaced by a simply closed rectifiable curve which encloses the part of $\sigma(F)$ with $\Re(\lambda) \geq 1$ and separates it from the part of $\sigma(F)$ with $\Re(\lambda)<1$. It remains to prove theorem 2.1 since this theorem implies theorem 2.2. Let $f_{0}=x-F_{0}$ where $F_{0}$ satisfies the assumptions of our theorem 3.2. If $\Re(\lambda)=1$ contains no point of $\sigma\left(F_{0}\right)$ no new proof is required. Otherwise, $\sigma\left(F_{0}\right)$ contains a: finite number of points on $\Re(\lambda)=1$ which we denote by $v_{1}, v_{2}, \ldots, v_{q}$. As in the proof of lemma 3.5 let $\mu_{1}, \bar{\mu}_{1}, \ldots, \bar{\mu}_{r}, \bar{\mu}_{r}$ be the distinct complex, and $\bar{\lambda}_{1}, \ldots, \lambda_{p}$ the distinct real eigenvalues of $F$ with $\Re(\lambda)>1$ while $\sigma^{-}\left(F_{0}\right)$ denotes the set $\sigma\left(F_{0}\right) \cap\{\Re(\lambda)<1\}$. Let then $c_{i}, c_{i}^{\prime}, c_{i}^{\prime \prime}, c_{i}^{\prime \prime \prime}$ be circles with centers $\lambda_{i}, \mu_{i}, \bar{\mu}_{i}, \nu_{i}$ resp. of the following property: if $o_{i}, o_{i}^{\prime}, o_{i}^{\prime \prime}, o_{i}^{\prime \prime \prime}$ are the open disks bounded by these circles then the closures of these disks are disjoint; for each of these closures its center is the only point belonging to $\sigma\left(F_{0}\right)$, and those of these closures whose centers are not on the real axis don't intersect the real axis. Let then $0^{-}$be an open set in $\Re(\lambda)<1$ containing $\sigma^{-}\left(F_{0}\right)$ and 0 be the union of $0^{-}$and all the open disks defined above. Then there exists an $\varepsilon$ such that

$$
\left\|F-F_{0}\right\|<\varepsilon
$$

implies

$$
\left.\dot{\sigma}(F)<0^{18}\right) .
$$

\footnotetext{
17) However (because of assumption $\left.A^{\prime}\right) \lambda=1$ cannot be an eigenvalue.

18) Cf. footnote ${ }^{10}$ ).
} 
By lemma 2.2 it is possible to choose $\varepsilon$ in such a way that in addition to (3.18) the equality (2.11) holds with the $m_{i}(F)$ denoting the dimensions of the ranges of the projections (2.10) wher the $c_{i}$ are the above circles with the real centers $\lambda_{i}$. Now by the choice of $\varepsilon$ the intersection $\sigma(F) \cap\{\Re(\lambda) \geq 1\}$ is contained in the disks $o_{i}, o_{i}^{\prime}, o_{i}^{\prime \prime}, o_{i}^{\prime \prime \prime}$, and since by construction only the $o_{i}$ intersect the real axis, the real points of $\sigma(F) \cap\{\Re(\lambda) \geq 1\}$ are contained in the $o_{i}$. Since $F$ is real the points of $\sigma(F) \cap\{\Re(\lambda) \geq 1\}$ contained in $o_{i}$ may be written $\lambda_{1}^{i}, \ldots, \lambda_{p_{i}}^{i}, \mu_{1}^{i}, \overline{\mu_{1}^{i}}, \ldots, \mu_{r_{i}}^{i}, \overline{\mu_{\psi_{i}}^{i}}$ where the $\lambda$ 's are real and the $\mu^{\prime}$ s non real. To each of these points corresponds in the usual way a projection and we denote the dimensions of the ranges of these projections by $m_{j}^{i}(F)$, $n_{j}^{i}(F), \bar{n}_{j}^{i}(F)$. Then an argument similar to the one leading to (2.14) shows that

$$
m_{i}(F)=\sum_{j=1}^{p_{i}} m_{j}^{i}(F)+\sum_{j=1}^{r_{i}}\left(n_{j}^{i}(F)+\bar{n}_{j}^{i}(F)\right) .
$$

But $n_{j}^{i}(F)=\bar{n}_{j}^{i}(F)$ since $F$ is real. Therefore we see from (3.19) and (2.11) that

$$
m_{i}\left(F_{0}\right) \equiv \sum_{j=1}^{p_{i}} m_{j}^{i}(F) \bmod 2 .
$$

By theorem 3.1 this equality implies (2.7).

REMARK 3.1. If $f(x)=x-F(x)$ is linear, non singular, real, and $F(x)$ completely continuous, then the assumptions $A^{\prime}, B^{\prime}, C^{\prime}$ follow. For the restriction of $f$ to $R$ (cf. lemma 3.1) an index $j_{1}(f)$ can then be defined by Leray-Schauder's method (cf. [6], section 11). It is easily seen that $j_{1}(f)=j(f)$ if one observes that the "degree $m_{i}$ in the sense of Goursat" used in [6] is identical with the number $m_{i}$ introduced in Assumption $C$ of the present paper, and if one uses the "Conclusion" in $[6]$, p. 58.

\section{HOMOTOPY PROPERTIES. THE PRODUCT THEOREM.}

Definition 4.1. Let $F, G$ be bounded linear real transformations mapping the complex Banach space $X$ into itself and satisfying the assumptions $A^{\prime}$, $B^{\prime}, C^{\prime}$ of section $3 . F$ and $G$ are called homotopic if for each $t$ in the interval $0 \leqq t \leqq 1$ there exists an $F_{t}$ which satisfies all assumptions just mentioned for $F$ and $G$, which is moreover continuous in $t$ and such that $F_{0}=F, F_{1}=G$. The maps

$$
f(x)=x-F(x), \quad g(x)=x-G(x)
$$

are called homotopic if $F$ and $G$ are homotopic.

THEOREM 4.1. Let the linear bounded maps $F$ and $G$ be real and satisfy the assumptions $\mathrm{A}^{\prime}, \mathrm{B}^{\prime}, \mathrm{C}^{\prime}$. Then the maps (4.1) are homotopic if and only if $j(f)=j(g)$. In particular: $j(f)=+1$ if and only if $f$ is homotopic to the identity map, and $j(f)=-1$ if and only if $f$ is homotopic to a map $g(x)$ of the form $g(x)=x-2 \varphi(x) x^{0}$ where $x^{0}$ is an arbitrarily chosen real element of $X$ of norm 1 , and $\varphi(x)$ an arbitrary linear real functional with $\varphi\left(x^{0}\right)=1$. 
Proof. That $j(f)=+1$ if $f$ is homotopic to the identity, and $j(f)=-1$ if $f=g$ is a consequence of theorem 3.1 since the index of the identity map is +1 , and $j(g)$ is easily seen to be -1 . Before proceeding to prove the converse we need a few simple facts which we state as lemmas.

LemMa 4.1. Let $X^{0}$ be a finite dimensional subspace of $X, \widetilde{F}^{0}$ a real linear map of $X^{0}$ into itself and $I^{0}$ the identity map of $X^{0}$. The eigenvalues $\lambda_{1}, \lambda_{2}, \ldots, \lambda_{n}$ of $\widetilde{F}^{0}$ are supposed to be all real and $>1$. Then $f^{0}=I^{0}-\widetilde{F}^{0}$ is homotopic to $-I^{0}$.

Proof. We choose the coordinate system in $X^{0}$ in.such a way that the matrix $M$ representing $\widetilde{F}_{0}$ in this coordinate system is in the Jordan normal form, i.e., that $M$ is direct sum of matrices each of which contains one of the $\lambda_{i}$ in the main diagonal and (unless it is one dimensional) contains one's directly above the main diagonal while all its other elements are zero. We define for $0 \leq t \leq 1$ as $M_{t}$ the matrix obtained from $M$ by replacing each $\lambda_{i}$ by $\lambda_{i}(1-t)+2 t$ and the one's by $1-t$, and by $\widetilde{F}_{t}^{0}$ the transformation given by $M_{t}$. Then $f_{1}^{0}=I^{0}-\widetilde{F}_{1}^{0}$ has the matrix $I^{0}-2 I^{0}=-I^{0}$ and is homotopic to $f^{0}=I^{0}-\widetilde{F}_{0}^{0}$.

LEMMA 4.2. With the notations and assumptions of lemma 4.1 let $N$ be the dimension of $X^{0}$. Then: i) if $N$ is even, $f^{0}$ is homotopic to the identity $I^{0}$; ii) if $N$ is odd, $\tilde{f}^{0}$ is homotopic to a map of the form $g(x)=x-2 \varphi(x) x^{0}$ where $x^{0}$ is an arbitrarily chosen element of $X^{0}$ of norm 1 and $\varphi(x)$ a linear functional with $\varphi\left(x^{0}\right)=1$; iii) if $X^{0}$ is invariant under conjugation then $x^{0}$ and $\varphi(x)$ may be chosen to be real.

Proof. On account of lemma.4.1 we may assume that $\tilde{f}^{0}=-I^{0}$. Now it is well known that in a space of even dimension the negative identity map is homotopic to the identity map. Applying this fact to $X^{0}$ proves i), and applying it to the even dimensional space which together with $x^{0}$ spans $X^{0}$ proves obviously ii). Under the assumption made in iii), $x^{0}$ obviously can be chosen real ${ }^{19}$ ). We define $\varphi(x)$ first for $x=\alpha x^{0}$ by setting $\varphi(x)=\alpha$. Since $x^{0}$ is real $\varphi(x)$ is real. By the following lemma 4.3 we can extend $\varphi(x)$ to $X$ as a real linear functional.

Lemma 4.3. Let $X$ be a complex Banach space with a conjugation and $X_{1}$ a finite dimensional linear subspace which is invariant under this conjugation, and $l(x)$ a linear functional defined on $X_{1}$ which is real in the sense of definition 3.3. Then $l(x)$ can be extended to $X$ with the original bound $L$ as a real linear functional.

Proof. Let $R$ be the real Banach space consisting of the real elements of $X$ (cf. lemma 3.1). It is then easily seen that the intersection $R_{1}=R \cap X_{1}$ is a finite dimensional linear subspace of $R$. Therefore we can by the HahnBanach theorem for real Banach spaces extend $l(x)$ as a real valued linear functional to $R$ with the original bound $L$. Let now $x$ be an arbitrary element

\footnotetext{
19) Let $x \neq 0$ be an element of $X^{0}$. If then in the decomposition (3.5) $x^{\prime} \neq 0$ we set $x^{0}=(x+\bar{x}) /\|x+\bar{x}\|_{\text {. If }} x^{\prime}=0$ we replace $x$ by $i x$.
} 
of $X$. By lemma 3.2 we can write uniquely

We then define

$$
x=x^{\prime}+i x^{\prime \prime} \quad\left(x^{\prime}, x^{\prime \prime}<R\right) .
$$

$$
l(x)=l\left(x^{\prime}\right)+i l\left(x^{\prime \prime}\right) \text {. }
$$

It is a matter of simple computation to verify first the additivity of $l(x)$ and then the equality $l(\alpha x)=\alpha l(x)$ for arbitrary complex $\alpha$. To prove the boundedness of $l(x)$ with the same bound $L$, let

Then

$$
l(x)=|l(x)| e^{-i \vartheta}, \quad y=x e^{i \vartheta}=y^{\prime}+i y^{\prime \prime} \quad \text { with } y^{\prime}, y^{\prime \prime}<R .
$$

$$
|l(x)|=l\left(x e^{i, y}\right)=l(y)=l\left(y^{\prime}\right)+i l\left(y^{\prime \prime}\right) .
$$

Since $l\left(y^{\prime}\right), l\left(y^{\prime \prime}\right)$ are real numbers and $y^{\prime}<R$, this implies

$$
|l(x)|=l\left(y^{\prime}\right) \leqq L\left\|y^{\prime}\right\|=L\|y+\ddot{y}\| / 2 \leqq L \frac{\|y|+| \bar{y}\|}{2}=L\|y\|=L\|x\| .
$$

Finally, it is immediately verified that $l(x)$ is real, i.e., satisfies $l(\bar{x})=\overline{l(x)}$.

Lemma 4.4. Let $X$ be the direct sum of the subspaces $X^{\mathbf{0}}$ and $X^{\mathbf{1}}$, and $E^{\mathbf{0}}$ and $E^{1}$ the projections of $X$ onto $X^{0}$ and $X^{1}$ resp. Let $G^{i}$ be a bounded linear map of $X^{i}$ into itself $(i=0,1)$. Detine for $x<X$ the map $G(x)=G^{0} E^{0}(x)+$ $G^{1} E^{1}(x)$. Then $\sigma(G)$ is the union of $\sigma\left(G^{0}\right)$ and $\sigma\left(G^{1}\right)$.

Proof. Since for any bounded transformation $T$ the spectrum $\sigma(T)$ and the resolvent set $\varrho(T)$ are complimentary sets (with respect to the whole $\lambda$ plane) the statement of the lemma is equivalent to the equality

$$
\varrho(G)=\varrho\left(G^{0}\right) \cap \varrho\left(G^{1}\right) \text {. }
$$

But this equality is easily derived from the fact the equation $\lambda x-G(x)=y$ is equivalent to the pair of equations for $x^{0}=E^{0} x$ and $x^{1}=E^{1}(x)$

$$
\lambda x^{0}-G^{0}\left(x^{0}\right)=E^{0}(y), \quad \lambda x^{1}-G^{1}\left(x^{1}\right)=E^{1}(y)
$$

together with the theorem ${ }^{20}$ ) that an everywhere defined inverse of an everywhere defined bounded linear transformation is itself bounded.

We now return to the proof of theorem 4.1. Let $\Gamma^{0}$ and $\Gamma^{1}$ be two simply closed rectifiable and disjoint curves oriented in the counter clockwise sense of the following properties: i) they lie in the resolvent set $\varrho(F)$ of $F$; ii) the open domains $D^{0}$ and $D^{1}$ bounded by them are disjoint; iii) let $\sigma^{0}$ be the part of $\sigma(F)$ which is real and $>1$; then there exists an interval of the real axis which contains the point $\lambda=2$ and $\sigma^{0}$, and which itself is contained in $D^{0}$; iv) $\sigma^{1}=\sigma(F)-\sigma^{0}$ lies in $D^{1}$. We set

$$
\begin{aligned}
& F^{0}=\frac{1}{2 \pi \sqrt{-1}} \int_{I^{0}} \mu R_{\mu}(F) d \mu, \quad F^{1}=\frac{1}{2 \pi \sqrt{-1}} \int_{\Gamma^{1}} \mu R_{\mu}(F) d \mu \\
& E^{0}=\frac{1}{2 \pi V-1} \int_{\Gamma^{0}} R_{\mu}(F) d \mu, \quad E^{1}=\frac{1}{2 \pi \sqrt{ }-1} \int_{\Gamma^{1}} R_{\mu}(F) d \mu \\
& \text { (4.4). } X^{0}=E^{0} X \text {, } \\
& X^{1}=E^{1} X \text {. }
\end{aligned}
$$

20) See e.g. [4], p. 29, Corollary. 
Then, as well known ${ }^{21}$ ), $F^{0}$ leaves $X^{0}, F^{1}$ leaves $X^{1}$ invariant, the restriction $\widetilde{F}^{0}$ of $F^{0}$ to $X^{0}$ has $\sigma^{0}$ as its spectrum, and $X$ is the direct sum of $X^{0}$ and $X^{1}$. $\sigma^{0}$ consists by assumption of a finite number of points $\lambda_{1}, \ldots, \lambda_{n}$. If $m_{i}$ is the dimension of the range of the projection (2.1) and $N$ the dimension of $X^{0}$ comparison of (2.1) with $(4.3)$ and (4.4) shows that $N=\sum_{i=1}^{n} m_{i}$. Consequently,
we see from theorems $3.1,3.2$ that

$$
j(f)=(-1)^{N}
$$

Now $X^{0}$ is invariant under conjugation by lemma 3.6 and $\widetilde{F}^{0}$ satisfies the assumptions of lemma $4 \cdot 2^{22}$. Therefore there exists for $0 \leq t \leq 1$ a map $\widetilde{F}_{t}^{0}$ of $X^{0}$ into itself (satisfying $\mathrm{A}^{\prime}, \mathrm{B}^{\prime}, \mathrm{C}^{\prime}$ ) such that $\widetilde{F}_{0}^{0}=\widetilde{F}^{0}$ while

$$
\tilde{f}_{1}^{0}(x)=x-\widetilde{F}_{1}^{0}(x)= \begin{cases}x & \text { if } N \text { is even } \\ x-2 \varphi(x) x^{0} & \text { if } N \text { is odd }\end{cases}
$$

where $x^{0}, \varphi$ are as in lemma 4.2. Let now $\widetilde{F}^{1}$ be the map $F^{1}$ [defined in (4.2)] restricted to $X^{1}$, and $\widetilde{F}_{t}^{1}=(1-t) \widetilde{F}^{1}$ for $0 \leqq t \leqq 1$. The spectrum $\sigma\left(\widetilde{F}_{t}^{1}\right)$ of $\widetilde{F}_{t}^{1}$ is obtained in multiplying the points of $\sigma\left(\widetilde{F}^{1}\right)$ by $1-t$, and since the real points of $\sigma\left(\widetilde{F}^{1}\right)=\sigma\left(F^{1}\right)$ are in the half plane $R(\lambda)<1$ and $\widetilde{F}^{1}$ satisfies $\mathrm{A}^{\prime}, \mathrm{B}^{\prime}, \mathrm{C}^{\prime}$; it follows easily that also $\widetilde{F}_{t}^{1}$ satisfies $\mathrm{A}^{\prime}, \mathrm{B}^{\prime}, \mathrm{C}^{\prime}$. We now define for arbitrary $x$ in $X$

$$
F_{t}(x)=\widetilde{F}_{t}^{0} E^{0}(x)+\widetilde{F}_{t}^{1} E^{1}(x) .
$$

Since by lemma 4.4; $\sigma\left(F_{t}\right)$ is the union of $\sigma\left(\widetilde{F}_{t}^{0}\right)$ and $\left(\widetilde{F}_{i}^{1}\right)$ it follows that $F_{t}$ satisfies assumptions $\mathrm{A}^{\prime}, \mathrm{B}^{\prime}, \mathrm{C}^{\prime}$, and $f(x)=x-F(x)=x-F_{0}(x)$ is by $(4.6)$ seen to be homotopic to

$$
\begin{array}{rlrl}
f_{1}(x) & =x-F_{1}(x)=\left(E^{0}(x)-\widetilde{F}_{1}^{0}(x)\right)+E^{1}(x)=\tilde{f}_{1}^{0} E^{0}(x)+E^{1}(x) \\
& =x & \text { if } N \text { is even } \\
& =x-2 \varphi(x) x^{0} \quad \text { if } N \text { is odd. }
\end{array}
$$

It remains to prove that $x^{0}$ and $\varphi(x)$ can be chosen arbitrarily subject to the conditions of reality and to

$$
\left\|x^{0}\right\|=1, \quad \varphi\left(x^{0}\right)=1,
$$

i.e., we have to show: if

$$
f(x)=x-2 \varphi(x) x^{0}, \quad g(x)=x-2 \psi(x) y^{0}
$$

where $x^{0}, y^{0}$ are real elements of $X$ and $\varphi(x), \psi(x)$ real linear functionals satisfying (4:7) and

$$
\left\|y^{0}\right\|=1, \quad \psi\left(y^{0}\right)=1,
$$

then $f$ and $g$ are homotopic. We distinguish two cases:

CASE 1. $x^{0}$ and $y^{0}$ are linearly dependent. In this case $y^{0}=\varepsilon x^{0}$ where because of (4.7), (4.8), $\varepsilon$ is a complex number of absolute value 1 . Then

21) $[1]$, p. 197 .

${ }^{22}$ ) That $\widetilde{F^{0}}$ is real follows from the assumption that $F$ is the real since $\widetilde{F^{0}}$ is the restriction of $F$ to $X^{0}$. 
$\psi(x) y^{0}=\widetilde{\psi}(x) x^{0}$ if $\tilde{\psi}(x)$ is defined as $\psi(\varepsilon x)$, and $\tilde{\psi}\left(x^{0}\right)=\psi\left(\varepsilon x^{0}\right)=\psi\left(y^{0}\right)=1$, which shows that we may assume that $\varepsilon=1$, i.e., $y^{0}=x^{0}$. With this assumption we set $\varphi_{t}(x)=(1-t) \varphi(x)+t \psi(x)$ for $0 \leqq t \leqq 1$. We note that $\varphi_{t}\left(x^{0}\right)=1$. and that therefore the spectrum of $F_{t}(x)=2 \varphi_{t}(x) x^{0}$ consists of the points $\lambda=0$ and $\lambda=2$, and that the range of the projection belonging to $\lambda=2$ is of dimension 1. Therefore $F_{t}(x)$ satisfies $\mathrm{A}^{\prime}, \mathrm{B}^{\prime}, \mathrm{C}^{\prime}$. Moreover, with $f_{t}(x)=$ $x-F_{t}(x)$ we have $t_{0}=f, f_{1}=g$. Thus $f$ and $g$ are homotopic in the sense of definition 4.1 .

CASE 2. $x^{0}$ and $y^{0}$ are linearly independent. Let $X^{2}$ be the subspace of $X$ spanned by $x^{0}$ and $y^{0}$, and $E^{2}$ a real projection with range $X^{223}$. Let for $x<X^{2}, \tilde{r}_{t}(x)$ be a rotation about the origin such that $\left.\tilde{r}_{0}\left(x^{0}\right)=x^{0}, \tilde{r}_{1}\left(x^{0}\right)=y^{0}{ }^{24}\right)$, let $r_{t}=\tilde{r}_{t} /\left\|\tilde{r}_{t}\right\|$, and let the extension of $r_{t}$ to all of $X$ be defined by

Moreover, set

$$
r_{t}(x)=x-E^{2}(x)+r_{t}\left(E^{2}(x)\right) \text {. }
$$

$$
\varphi_{i}(x)=\varphi\left(r_{t}^{-1}(x)\right), \quad F_{t}(x)=2 \varphi_{t}(x) r_{t}\left(x^{0}\right) .
$$

If one observes that $p_{t}\left(r_{t}\left(x^{0}\right)\right)=\varphi\left(x^{0}\right)=1$, it is easily seen that the spectrum of $F_{t}$ consists of the points $\lambda=0$ and $\lambda=2$, and that $F_{t}$ satisfies $\mathrm{A}^{\prime}, \mathrm{B}^{\prime}, \mathrm{C}^{\prime}$. Therefore setting $f_{t}(x)=x-F_{t}(x)$ shows that $f(x)$ is homotopic to $h(x)=$ $x-2 \varphi_{1}(x) y^{0}$ since $r_{1}\left(x^{0}\right)=y^{0}$. To see finally that $h$ is homotopic to $g$ we have only to notice that we are now in case ${ }^{25}$ ).

LeMMa 4.5. Let $X$ be a complex Banach space with a conjugation, and $X_{p}$ a p-dimensional linear subspace which is invariant under this conjugation $(p$ a finite integer). Then there exists a real projection $E_{p}$ of $X$ whose range is $X_{p}$ (cf. definition 3.3).

Proof. Without the reality requirement $E_{p}$ may, according to Mackey ${ }^{26}$ ), be constructed as follows: let $x_{1}, x_{2}, \ldots, x_{p}$ be a base for $X_{p}$. Then for $j=1, \ldots, p$ the coefficient $f_{j}(x)$ of $x_{j}$ in the representation of an element $x$ of $X$ as linear combination of $x_{1}, \ldots, x_{p}$ is a linear functional on $X_{p}$ which may be extended to $X$. Calling the extension again $f_{j}(x)$, it turns out that $E_{p}(x)=\sum_{j=1}^{p} x_{j} f_{j}(x)$ is a projection with range $X_{p}$. We will now show that with a proper choice of the basis $x_{1}, \ldots, x_{p}, E_{p}$ is real. We will prove first that it is possible to construct a basis of real elements: let $x \neq 0$ be an element of $X_{p}$; then by assumption $\bar{x}<X_{p}$, and therefore $x+\bar{x}$ and $(x-\bar{x}) / i$ are real element of $X_{p}$ at least one of them not being the zero element. Thus if $q$ denotes the maximal number of real elements of $X_{p}$ which are linearly independent (with respect to complex numbers) then $q \geq 1$. We will prove $q=p$ : let $r_{1}, r_{2}, \ldots, r_{q}$, be linearly independent real elements of $X_{b}$, and

23) Such $E^{2}$ exists by the following lemma 4.5.

24) i.e., if $x=\alpha^{0} x^{0}+\alpha^{\prime} y^{0}$ then $\tilde{r}_{t}(x)=\beta^{0} x^{0}+\beta^{\prime} y^{0}$ where $\beta^{0}=\alpha^{0} \cos (t \pi / 2)-\alpha^{\prime} \sin (t \pi / 2)$, $\beta^{\prime}=\alpha^{0} \sin (t / \pi / 2)+\alpha^{\prime} \cos (t \pi / 2)$.

25) Note that $\varphi_{1}\left(y^{0}\right)=\varphi_{1}\left(\gamma_{1}\left(x^{0}\right)\right)=\varphi\left(x^{0}\right)=1$.

26) [7], lemma 3.1 . 
$x=x^{\prime}+i x^{\prime \prime}\left(x^{\prime}, x^{\prime \prime}\right.$. real) an arbitrary element of $X_{p}$. It follows from these definitions that there exist constants $\alpha_{j}^{\prime}, \alpha_{j}^{\prime \prime}$ (which may be complex) such that

$$
x^{\prime}=\sum_{j=1}^{q} \alpha_{i}^{\prime} r_{j}, \quad x^{\prime \prime}=\sum_{j=1}^{q} \alpha_{j}^{\prime \prime} r_{j} .
$$

Then $x=x^{\prime}+i x^{\prime \prime}$ is a linear combinations of the $r_{j}$ which proves that the $r_{j}$ form a base and $q=p$. Thus we may assume that the elements $x_{1}, \ldots, x_{q}$ of the originat base are all real. But then comparison of

$$
\bar{x}=\sum_{j=1}^{q} f_{j}(\bar{x}) x_{i} . \text { with } \bar{x}=\overline{\sum_{j=1}^{q} f_{j}(x) x_{j}}=\sum_{j=1}^{q} \overline{f_{j}(x)} x_{j}
$$

shows that $f_{j}(\bar{x})=\overline{f_{j}(x)}$, i.e., that the functionals $f_{j}(x)$ are real. Therefore, by lemma 4.3 their extensions can also be constructed to be real, and the above constructed projection $E_{p}$ is then obviously also real.

REMarK 4.2. It is well known ${ }^{27}$ ) that the range $X_{\lambda_{i}}$ of the projection (2.1) is the space spanned by the solutions of the totality of equations $\left(\lambda_{i} I-F\right)^{n}=0$. for $n=1,2, \ldots$. If now $F$ and $\lambda_{i}$ is real then $X_{\lambda_{i}}$ is invariant under conjugation (lemma 3.5). It follows (as shown in the proof of lemma 4.5) that $X_{\lambda_{i}}$ can be spanned by elements of $R$, in other words, the dimension $m_{i}$ of $X_{\lambda_{i}}$ is the number of linearly independent solutions in $R$ of the above equations. It follows then from theorem 3.1 that the index $j(f)$ is determined if $F$ is known in $R$. If $F$ is in addition completely continuous it also follows that $j(f)$ agrees with the Leray-Schauder index of $f$ (cf. [6] p. 57, 58).

THEOREM 4.2. (Product theorem.) Let $f(x)=x-F(x)$ and $g(x)=x-G(x)$. Let $F(x), G(x)$, and $x-(f g)(x)$ be real and satisfy $\mathrm{A}^{\prime}, \mathrm{B}^{\prime},-\mathrm{C}^{\prime}$. Then

$$
j(f g)=j(f) j(g) \text {. }
$$

Proof. If one of the mappings, say $f$, has index +1 , then by theorem 4.1 $f$ is homotopic to the identity map and therefore $f g$ is homotopic to $g$. We have then, again using theorem 4.1, $j(f g)=j(g)=1 \cdot j(g)=j(f) j(g)$, which proves (4.9) in the case considered. Assume now

$$
j(f)=j(g)=-1 \text {. }
$$

Then by theorem 4.1, $f$ and $g$ are both homotopic to a map of the form

$$
h(x)=x-2 \varphi(x) x^{0} \quad \text { with } \quad\left\|x^{0}\right\|=\varphi\left(x^{0}\right)=1 .
$$

Then $f g$ is homotopic to $h^{2}$, and by theorem 4.1, we have $j(f g)=j\left(h^{2}\right)$. This equality together with (4.10) shows that for the proof of (4.9) it will be sufficient to show that

$$
j\left(h^{2}\right)=+1
$$

But (4.12) is certainly true since $h^{2}(x)=x$ as is easily verified from (4.11).

27) See e.g., [1], p. 198. 
5. NON LINEAR MAPS. Let $X$ be a complex Banach space, $D$ a bounded open set in $X, \bar{D}$ its closure, and $B=\bar{D}-D$ its boundary. We will define the mapping degree and prove the homotopy property for a class of "smooth" mappings of $\bar{D}$ into $X$.

Definition 5.1. A mapping

$$
f(x)=x-F(x)
$$

of $\bar{D}$ into $X$ is called smooth if it satisfies the following conditions: i) in each point $x$ of $\bar{D}, f(x)$ has a bounded Fréchet differential $\left.a(x, h)^{28}\right)$ which is uniformly continuous in $x$ in the sense that to each positive $\varepsilon$ there exists a $\delta$ such that

$$
\| d(x, h)-d\left(x_{0}, h\|<\varepsilon\| h \| \text { for }\left\|x-x_{0}\right\|<\delta\right.
$$

ii) $d(x, h)$ (as linear operator in $h$ ) has an inverse $d^{-1}(x, h)=l(x, h)$ with a bound which is uniform for all $x<\bar{D}$; iii) if the point $y$ of $X$ is not on the image of the boundary $B$ of $D$, then there are at most a finite number of points $x$ in $D$ which satisfy the equation

$$
f(x)=y \text {; }
$$

iv) the linear operator in $h, D(x, h)=h-d(x, h)$ satisfies the conditions $\mathrm{A}$, $\mathrm{B}, \mathrm{C}$ of section $2 ; \mathrm{v})$ the image of a closed set is closed.

REMARK 5.1. If only i) and ii) are assumed and $F(x)$ is completely continuous then v) is automatically satisfied; so is iii), for it can be easily seen that otherwise there would be a point $x$ in $D$ satisfying (5.3) in every neighborhood of which there would be other solutions of (5.3) which would be a contradiction to the lemma 5.1 below. If in addition $D(x, h)$ is completely continuous (in $h$ ) then iv) is also automatically satisfied.

Definition 5.2. Let $f(x)$ be a smooth mapping of $\bar{D}$ into $X$, and $y$ a point of $X$ which is not on the image of the boundary $B$ of $D$. We define the degree $j(f, y, D)$ of $f$ at $y$ with respect to. $D$ as follows: if $(5.3)$ has no solution $x$ in $D$ we set $j(f, y, D)=0$; if there are solutions in $D$ there are by assumption iii) of definition 5.1 only a finite number, say $x_{1}, x_{2}, \ldots, x_{p}$. By assumption iv) of definition 5.1 for each of the linear operators in $h, d\left(x_{0}, h\right)(\varrho=1, \ldots, r)$ the index $j_{0}=j\left(d\left(x_{0}, h\right)\right)$ is defined according to definition 2.1. Let $p$ be the number of those $j_{g}$ which are +1 , and $q$ the number of those which are -1 . Then we define

$$
\left.j(f, y, D)=p-q^{29}\right) .
$$

28) For fixed $x, d(x, h)$ is a bounded linear operator in $h$.

29) In the Leray Schauder theory the equality (5.4) is a theorem (cf. [6], p. 56). In finite dimensional spaces $p$ is the numbers of those $x$ for which the Jacobian of $f(x)$. is positive, and $\dot{q}$ the number of those for which it is negative. With this interpretation (5.4) is the basic definition used as starting point in Nagumo's theory for the degree in finite dimensional spaces. (See [8].) 
Definition 5.3. Let $f(x)=x-F(x), g(x)=x-G(x)$ be two smooth mappings of $\bar{D}$ into $X$. Then $f$ and $g$ are called homotopic if for each $t$ in the interval $0 \leqq t \leqq 1$ there exists a smooth map

$$
f_{t}(x)=x-F(x, t) \text { with } f_{0}(x)=f(x), \quad f_{1}(x)=g(x)
$$

where $F(x, t)$ is continuous in the product $I I=\bar{D} \times\{0 \leqq t \leq 1\}$, where the continuity in $t$ for fixed $x$ is uniform for $x<\bar{D}$, and where moreover the following two conditions are satisfied: a) the Fréchet differential $d(x, t, h)$ of $f_{t}(x)$ (with respect to $x$ ) is continuous in $x$ in the sense that to every positive $\varepsilon$ there exist positive numbers $\delta$ and $\tau$ such that

$$
\| d(x, t, h)-d\left(x_{0}, t_{0}, h\|<\varepsilon\| h \| \text { for }\left\|x-x_{0}\right\|<\delta, \quad\left|t-t_{0}\right|<\tau ;\right.
$$

b) the inverse $d^{-1}(x, t, h)=l(x, t, h)$ of $d(x, t, h)$ as linear operator in $h$ exists and is bounded uniformly on $\bar{D}$, i.e., there exists a positive $m$ such that

$$
\|l(x, t, h)\|<m\|h\| \text {. }
$$

THEOREM 5.1. Let $f, g$ be two smooth mappings of $\bar{D}$ into $X$, and $y$ a point of $X$ which does not lie on the image of the boundary $B$ of $D$ under $f$ or $g$. We assume that $f$ and $g$ are homotopic (Def. 5.3) and that moreover the map (5.5) can be chosen such that

$$
f_{t}(x) \neq y \quad \text { for } \quad x<B, \quad 0 \leqq t \leqq 1 .
$$

Then

$$
j(f, y, D)=j(g, y, D) \text {. }
$$

Proof. It will be obviously sufficient to show that $j\left(f_{t}, y, D\right)$ is.continuous in $t$. To do this we first prove

LEMMA $\left.5.1^{30}\right)$. For each $t$ in the unit interval let $f_{t}(x)=f(x, t)=x-F(x, t)$ be a.map of $\bar{D}$ into $X$ which satisfies conditions i) and ii) of definition 5.1 , is continuous on the product of $\bar{D}$ with the unit interval, and satisfies conditions a) and b) of definition 5.3. Let $\vartheta, \vartheta_{1}, a_{0}, b_{0}$, and $\tau$ be positive numbers which are chosen as follows:

$$
0<\vartheta<\vartheta_{1}<1
$$

$a_{0}$ is then chosen in such a way that

$$
\| d(x, t, h)-d\left(x_{0}, t, h\|<\| h \| \vartheta / m \text { for } \quad\left\|x-x_{0}\right\|<a_{0}\right.
$$

where $m$ is a number satisfying (5.6); this choice is possible by assumption i) of definition 5.1. For $b_{0}$ we then choose any number satisfying

$$
0<b_{0}<a_{0}\left(1-\vartheta_{1}\right) / m \text {. }
$$

Let $x_{0}$ be a point of $D$ and set

$$
f\left(x_{0}, t\right)=y_{0}(t) \quad(0 \leq t \leq 1) .
$$

30) Lemma 5.1 and its proof are essentially an adaptation to the present purpose of results and methods contained in [3]. The proof is here given for the sake of completeness and of the possibility of referring later on to some of the inequalities contained in it. 
Then choose $\tau>0$ in such a way that

$$
\left\|y_{0}(t)-y_{0}\left(t_{0}\right)\right\|<b_{0} / 2 \text { for }\left|t-t_{0}\right|<\tau .
$$

Finally let $y$ be any point of $X$ which satisfies

$$
\left\|y-y_{0}(t)\right\|<b_{0} \quad \text { for } \quad\left|t-t_{0}\right|<\tau .
$$

Then the equation for $x$

$$
y=f(x, t)
$$

has one and only one solution $x=x(y, t)$ which satisfies

$$
\left\|x(t, y)-x_{0}\right\|<a_{0} \quad\left(\left|t-t_{0}\right|<\tau\right)
$$

and this solution is continuous in the product $D \times\left\{\left|t-t_{0}\right|<\tau\right\}$.

Proof. Subtracting (5.12) from (5.15) and setting

$$
\left\{\begin{aligned}
& x-x_{0}=\xi, \quad y-y_{0}(t)=\eta, \\
& f(x, t)-f\left(x_{0}, t\right)-d\left(x_{0}, t, x-x_{0}\right)=R\left(x, x_{0}, t\right)
\end{aligned}\right.
$$

we see that the equation for $\xi$

$$
\eta(t)=d\left(x_{0}, t, \xi\right)+R\left(x_{0}+\xi, x_{0}, t\right)
$$

is equivalent to (5.15). Multiplying (5.18) by $d^{-1}=l$ and setting

$$
l(\eta)=\zeta, \quad l\left(R\left(x_{0}+\xi, x_{0}, t\right)\right)=-S\left(x_{0}, t, \xi\right)
$$

we obtain for $\xi$ the equation

$$
\xi=\zeta(t)+S\left(x_{0}, t, \xi\right)
$$

which is still equivalent to (5.15). In order to solve (5.20) by successive approximations we prove the following two inequalities:

$$
\|\zeta\|<a_{0}\left(1-\vartheta_{1}\right)
$$

if (5.14) is satisfied, and

$$
\left\|S\left(x_{0}, t, \xi^{\prime \prime}\right)-S\left(x_{0}, t, \xi^{\prime}\right)\right\|<\vartheta\left\|\xi^{\prime \prime}-\xi^{\prime}\right\|
$$

The proof of (5.21) is immediate since by (5.19), (5.6), (5.17), (5.14), and (5.11)

$$
\|\zeta\|<m\|\eta\|=m\left\|y-y_{0}(t)\right\|<m b_{0}<a_{0}\left(1-\vartheta_{1}\right)
$$

As to the proof of (5.22) we obtain from the definition (5.17) of $R$ using the fact that $d(x, t, \xi)$ is linear in its third argument

$$
\left\{\begin{aligned}
R\left(x_{0}+\xi^{\prime \prime}, x_{0}, t\right)-R\left(x_{0}+\xi^{\prime}, x_{0}, t\right) & \\
= & {\left[f\left(x_{0}+\xi^{\prime \prime}, t\right)-f\left(x_{0}+\xi^{\prime}, t\right)\right]-d\left(x_{0}, t, \xi^{\prime \prime}-\xi^{\prime}\right) . }
\end{aligned}\right.
$$


If we apply the mean value theorem ${ }^{31}$ ) to the difference in the bracket, the right member of (5.24) may be written as

$$
\int_{0}^{1}\left\{d\left(x_{0}+\xi^{\prime}+\beta\left(\xi^{\prime \prime}-\xi^{\prime}\right), t, \xi^{\prime \prime}-\xi^{\prime}\right)-d\left(x_{0}, t, \xi^{\prime \prime}-\xi^{\prime}\right)\right\} d \beta .
$$

Since (5.23) implies the corresponding inequality for $\xi^{\prime}+\beta\left(\xi^{\prime \prime}-\xi^{\prime}\right)$ if $0 \leq \beta \leq 1$, we see from (5.10) (with $h$ replaced by $\xi^{\prime \prime}-\xi^{\prime}$ ) that the norm of the above integral is $<\left\|\xi^{\prime \prime}-\xi^{\prime}\right\| 9 / m$. Therefore using (5.6) and the definition (5.19) of $S$ we see that

$$
\begin{aligned}
\left\|S\left(x_{0}, t, \xi^{\prime \prime}\right)-S\left(x_{0}, t, \xi^{\prime}\right)\right\|= & \left\|l\left\{R\left(x_{0}+\xi^{\prime \prime}, x_{0}, t\right)-R\left(x_{0}+\xi^{\prime}, x_{0}, t\right)\right\}\right\| \\
& <m\left\|R\left(x_{0}+\xi^{\prime \prime}, x_{0}, t\right)-R\left(x_{0}+\xi^{\prime}, x_{0}, t\right)\right\| \\
& <m\left\|\xi^{\prime \prime}-\xi^{\prime}\right\| \vartheta / m
\end{aligned}
$$

which proves (5.22). As a special case we note that the inequality

$$
\left\|S\left(x_{0}, t, \xi\right)\right\|<\vartheta\|\xi\|
$$

is obtained from (5.22) by setting $\xi^{\prime \prime}=\xi, \xi^{\prime}=0$ since $S\left(x_{0}, t, 0\right)=0$ as is seen from (5.19) and (5.17).

Now from the inequalities (5.21), (5.22), and (5.25) one proves in the wellknown fashion (see, e.g., [3]) that the iterations

$$
\xi_{1}=\zeta, \quad \xi_{n+1}=\zeta+S\left(x_{0}, t, \xi_{n}\right) \quad(n=1,2, \ldots)
$$

are well defined (for $\zeta$ and $t$ in the domains specified) and satisfy the inequalities

$$
\left\|\xi_{n}\right\|<a_{0}\left(1-\vartheta_{1}\right) /(1-\vartheta), \quad\left\|\xi_{n}-\xi_{n-1}\right\|<a_{0} \vartheta^{n-1}
$$

which show that $\xi=\xi(\zeta, t)=\lim _{n \rightarrow \infty} \xi_{n}$ exists uniformly and that

$$
\|\xi\| \leq a_{0}\left(1-\vartheta_{\mathbf{1}}\right) /(1-\vartheta)<a_{0} .
$$

Obviously, $\xi$ satisfies (5.20), and $x=x(y, t)$ obtained from $\xi=\xi(\zeta, t)$ by (5.17) and (5.19) satisfies (5.15). The continuity of $x(y, t)$ follows from the uniformity of the convergence since each iteration $\xi_{n}$ is continuous in $(\zeta, t)$. The uniqueness of $\xi$ as solution of (5.20) follows in the usual way from (5.22) and (5.9).

We now return to the proof of theorem 5.1. We have to show: if $y=y_{1}$ is a point of $X$ for which (5.7) is satisfied then there exists a $\tau>0$ such that

$$
j\left(t_{t}, y_{1}, D\right)=j\left(t_{t_{0}}, y_{1}, D\right) \quad \text { for } \quad\left|t-t_{0}\right|<\tau \text {. }
$$

We distinguish two cases:

CASE 1. $t_{t_{0}}$ maps no point of $D$ on $y_{1}$. Then by $(5: 7)$ no point of the closed set $\bar{D}$ is mapped on $y_{1}$ by $t_{t_{0}}$, and it follows from assumption v) of definition 5.1 that the image under $t_{t_{0}}$ of $\bar{D}$ has a positive distance from $y_{1}$. This again implies by definition 5.3 that for small enough $\left|t-t_{0}\right|, y_{1}$ is not image point of $\bar{D}$ under the map $t_{t}$. Therefore for such $t(5.26)$ is true, both members of the equation being zero by definition $\mathbf{5 . 2}$.

31) [2].

Mathematische Zeitschrift. Bd. 63. 
CASE 2. $y_{1}$ is image point of $D$ under the map $t_{t_{0}}=f\left(x, t_{0}\right)$. Then by assumption iii) of definition 5.1 there are only a finite number of points in $D$, say $x^{1}, x^{2}, \ldots, x^{r}$ in $D$ such that

$$
f\left(x^{\varrho}, t_{0}\right)=y_{1} \quad(\varrho=1,2, \ldots, r) .
$$

Now by theorem 2.1 there exists an $\varepsilon>0$ such that any bounded linear operator $l^{e}(\xi)$ which satisfies the inequality

$$
\left\|d\left(x^{e}, t_{0}, \xi\right)-l^{e}(\xi)\right\|<\varepsilon\|\xi\|
$$

satisfies also the equality

$$
j\left(d^{e}, t_{0}, \xi\right)=j\left(l^{e}(\xi)\right) .
$$

In order to construct the required $\tau$ we remark first that by assumption a) of definition 5.3 it is possible to choose two positive numbers $\tau_{1}$ and $\delta$ such that

$$
\left\|d\left(x^{0}, t_{0}, \xi\right)-d(x, t, \xi)\right\|<\varepsilon\|\xi\|
$$

if

$$
\left|t-t_{0}\right|<\tau_{1}
$$

and

$$
\left\|x-x^{e}\right\|<\delta .
$$

We now chosose a number $a_{0}$ which satisfies the following requirements: i) (5.10) is satisfied with $x_{0}=x^{e}$; ii) we have

$$
\left|a_{0}\right|<\delta ;
$$

iii) the $r$ solid spheres with centers $x^{e}$ and radius $a_{0}$ are disjoint and contained in $D$. We then choose a number $b_{0}$ subject to the restriction (5.11), and a number $\tau_{2}$ such that

$$
\| f\left(x^{\varrho}, t\right)-f\left(x^{Q}, t_{0} \|<b_{0} / 2\right.
$$

if

$$
\left|t-t_{0}\right|<\tau_{2} .
$$

We finally choose a positive number $\tau_{3}$ as follows: let $C$ be the closure of the set obtained from $D$ by cutting out the spheres with centers $x^{\circ}$ and radius $a_{0}$, and denote by $f_{t}(C)$ the image of $C$ under the map $f_{t}$. Then $f_{t_{0}}(C)$ does not contain $y_{1}$. Therefore by assumption v) of definition 5.1, $f_{t_{0}}(C)$ has a positive distance from $y_{1}$. Consequently, there exists a $\tau_{3}$ such that $f_{t}(C)$ does not contain $y_{1}$ for $\left|t-t_{0}\right|<\tau_{3}$; we can then conclude that for some $\varrho$ of the set $1,2, \ldots, r$

$$
\left\|x-x^{0}\right\|<a_{0} \text { if } f(x, t)=y_{1} \text { and }\left|t-t_{0}\right|<\tau_{3} .
$$

We claim that (5.26) holds with $\tau=\min \left(\tau_{1}, \tau_{2}, \tau_{3}\right)$. Indeed, consider $f_{t}=f(x, t)$ for a $t$ in $\left|t-t_{0}\right|<\tau$. Let

$$
f\left(x^{e}, t\right)=y^{e}(t)
$$


With the choic of the constants $a_{0}, b_{0}, \tau$ made we can apply lemma 5.1 with $x_{0}=x^{e}$ and $y_{0}(t)=y^{e}(t)$ and see that the equation

$$
f(x, t)=y
$$

has for all $y$ satisfying

$$
\left\|y-y^{e}(t)\right\|<b_{0}
$$

one and only one solution $x^{\varrho}(y, t)$ which satisfies the inequality

$$
\left\|x^{e}(y, t)-x^{e}\right\|<a_{0} .
$$

Now by (5.34), (5.27), and (5.37), $y=y_{1}=f\left(x^{0}, t_{0}\right)$ satisfies (5.39). Therefore the equation for $x$

$$
f(x, t)=y_{1}
$$

has one and only one solution $x_{\mathbf{1}}^{e}=x^{e}\left(y_{1}, t\right)$ in (5.40). Then, by (5.33), $x=x_{\mathbf{1}}^{e}$ satisfies (5.32). Consequently, since $t$ satisfies (5.31) we see that $d\left(x_{1}^{e}, t, \xi\right)$ satisfies (5.30), i.e., that $(5.28)$ is true with $l^{o}(\xi)=d\left(x_{\mathbf{1}}^{0}, t, \xi\right)$, and gives us the equality

$$
j\left(d\left(x^{e}, t_{0}, \xi\right)\right)=j\left(d\left(x_{1}^{e}, t, \xi\right)\right) .
$$

Since by (5.36) $x_{1}^{1}, x_{1}^{2}, \ldots x_{1}^{r}$, are the only solutions in $D$ of (5.41) we obtain by definition 5.2 of the degree the desired equality (5.26) by summing (5.42) over $\varrho$.

THEOREM 5.2. Let $y=f(x)$ be a smooth mapping of the closure of the open bounded domain $D$ of $X$ into $X$, and $y_{0}$ a point of $X$ which is not situated on the image of the boundary $B$ of $D$. Then there exists a positive number $b_{0}$ such that

$$
j(f, y, D)=j\left(f, y_{0}, D\right) \text { for }\left\|y-y_{0}\right\|<b_{0} .
$$

Proof. We detinguish two cases. Case 1. No point of $D$ is mapped on $y_{0}$. Then it is easily seen from assumption v) of definition 5.1 that for small enough $\left\|y-y_{0}\right\|$, the point $y$ is also not in the image of $D$ or $B$. Therefore for such $y$ equation (5.43) is true, both members of the equality being zero.

CASE 2. Some points of $D$ are mapped on $y_{0}$. In this case let $x_{0}^{1}, x_{0}^{2}, \ldots, x_{0}^{r}$ be the points of $D$.which are mapped on $y_{0}$ : We choose a number $a_{0}$ which satisfies the following conditions: i) (5.10) is satisfied with $x_{0}=x_{0}^{\varrho}(\varrho=1,2, \ldots, r)$; ii) the equality

$$
j\left(d\left(x_{0}^{0}, \xi\right)\right)=j(d(x, \xi)) \text { for }\left\|x-x_{0}^{e}\right\|<a_{0} .
$$

holds for $\varrho=1,2, \ldots, r$, this choice being possible on account of (5.2) and theorem 2.1; iii) the spheres with centers $x_{0}^{\circ}$ and radius $a_{0}$ are disjoint and lie in $D$. We then choose a number $b_{0}$ subject to the following conditions: i) (5.11) is satisfied; ii) the sphere with radius $b_{0}$ about $y_{0}$ does not intersect the image of $B^{\prime}$ which choice is possible by assumption v) of definition 5.1; iii) let $C$ be the closure of the complement in $D$ of the union of the spheres $\left\|x-x_{0}^{0}\right\|<a_{0}$; the image of $C$ does not contain $y_{0}$ and has therefore (by the 
assumption just mentioned) a positive distance from $y_{0}$; we choose $b_{0}$ less than this distance. We claim that (5.43) is satisfied with this choice of $b_{0}$. Indeed, if $\left\|y-y_{0}\right\|<b_{0}$ then the equation $f(x)=y$ can have a solution only in one of the spheres $\left\|x-x_{0}^{0}\right\|<a_{0}$, and by lemma 5.1 ${ }^{32}$ ) it has exactly one, say $x=x^{o}$, in each of these spheres. Then (5.44) holds for $x=x^{0}$, and it follows from definition 5.2 that we obtain (5.43) by summing (5.44) (with $x$ replaced by $x^{\varrho}$ ) over $\varrho$.

6. REAL BANACH SPACES. The preceding sections dealt with complex Banach spaces. However, it is easy to carry over the theory from such spaces to real Banach spaces by imbedding them into a complex Banach space as will be done in this section.

Definition 6.1. We say that the real Banach space $R$ is imbedded in the complex Banach space $X$ if $X$ has a conjugation (def. 3.1) of the following property: let $R_{1}$ be the Banach space over the reals which is formed by the elements of $X$ which are invariant under this conjugation ${ }^{33}$ ); then $R$ and $R_{1}$ are algebraically isomorphic and isometric.

Lemma 6.1. Every real Banach space $R$ can be imbedded in a complex Bainach space.

Proof. We construct $X$ as follows: the elements $x$ of $X$ are the ordered couples $x=\left(x^{\prime}, x^{\prime \prime}\right)$ where $x^{\prime}, x^{\prime \prime}$ are elements of $R$. If $x=\left(x^{\prime}, x^{\prime \prime}\right)$ and $y=\left(y^{\prime}, y^{\prime \prime}\right)$ are two elements of $X$ and $\alpha=\alpha^{\prime}+i \alpha^{\prime \prime}$ a complex number we define the linear operations by

$$
x+y=\left(x^{\prime}+y^{\prime}, x^{\prime \prime}+y^{\prime \prime}\right), \quad \alpha x=\left(\alpha^{\prime} x^{\prime}-\alpha^{\prime \prime} x^{\prime \prime}, \alpha^{\prime} x^{\prime \prime}+\alpha^{\prime \prime} x^{\prime}\right) .
$$

Finally, following Krein and Gelfand [I. Gelfand, Normierte Ringe, Recueil Math., T. 9 (51), 1941, 3-23, §8] we define the norm $\|x\|$ of the element $x=\left(x^{\prime}, x^{\prime \prime}\right)$ by $\|x\|=\sup _{p}\left\|x^{\prime} \cos \varphi+x^{\prime \prime} \sin \varphi\right\|$.

DEFINITION 6.2. Let

$$
y=f(x)=x-F(x)
$$

be a linear non singular map of the real Banach space $R$ into itself with completely continuous $F(x)$. Denote by $\lambda_{1}, \lambda_{2}, \ldots, \lambda_{p}$ those eigenvalues of $F(x)$ which are real greater than $1^{34}$ ). Let $m_{i}$ be the number of linearly independent solutions in $R$ of the totality of equations $\left(\lambda_{i} x-F(x)\right)^{n}=0$ for $\left.n=i, 2, \ldots{ }^{35}\right)$. We then define the index $j(f)$ of $f$ by the equation

$$
j(f)=\sum_{i=1}^{p}(-1)^{m i}
$$

32) We apply lemma 5.1 with $f(x ; t) \equiv f(x)$.

33) Cf. Definition 3.2 and lemma 3.1.

34) That there are at most a finite number of eigenvalues $>1$ is a well known consequence of the complete continuity of $F$.

${ }^{35}$ ) It is well known that $m_{i}$ is finite. 
THEOREM 6.1. Let $F(x)$ satisfy the assumptions of definition 6.2. Then there exists $a \delta>0$ of the following property: if $g(x)=x-G(x)$ where $G(x)$ is completely continuous and

$$
\|f-g\|=\|F-G\|<\delta,
$$

then

$$
j(f)=j(g) .
$$

Proof. I et $X$ be the complex Banach space in which $R$ is imbedded by. lemma 6.1. By lemma 3.2 every element of $x$ of $X$ is of the form

$$
x=x^{\prime}+i x^{\prime \prime} \text { where } x^{\prime}=(x+\bar{x}) / 2 \text { and } x^{\prime \prime}=(x-\bar{x}) / 2 i \text { are in } R \text {. }
$$

We define

$$
\widetilde{F}(x)=F\left(x^{\prime}\right)+i F\left(x^{\prime \prime}\right), \quad \tilde{f}(x)=x-\widetilde{F}(x)
$$

and $\widetilde{G}, \tilde{g}$ correspondingly. It is then easily verified that $\widetilde{F}(x)$ is a linear completely continuous map of $X$ into itself and that the real points of its: spectrum coincide with the real eigenvalues of $F(x)$. Moreover, $\widetilde{F}(x)$ is real in the sense of definition 3.3 and satisfies the assumptions $A^{\prime}, B^{\prime}, C^{\prime}$ of section 3 (cf. remark 3.1). Now by remark 4.2 the numbers $m_{i}$ of definition 6.1 agree with those of theorem 3.1. Therefore

and similarly

$$
j(f)=j(\tilde{f})
$$

Now by theorem 3.2 there exists an $\varepsilon>0$ such that

$$
j(\tilde{f})=j(\tilde{g})
$$

if

$$
\|\widetilde{F}-\widetilde{G}\|<\varepsilon .
$$

We now set $\delta=\varepsilon / 2$ and claim that with this $\delta,(6.3)$ implies (6.4). Indeed, if we set $H=F-G$ and $\widetilde{H}=\widetilde{F}-\widetilde{G}$, we obtain, recalling (6.5) and (3.3)

$$
\begin{aligned}
\|\widetilde{F}(x)-\widetilde{G}(x)\| & =\|\tilde{H}(x)\|=\left\|H\left(x^{\prime}\right)+i H\left(x^{\prime \prime}\right)\right\| \leq\|H\|\left\{\left\|x^{\prime}\right\|+\left\|x^{\prime \prime}\right\|\right\} \\
& =\|H\|\{\|x+\bar{x}\|+\|x-\bar{x}\|\} / 2 \\
& \leqq\|H\|\{\|x\|+\|\bar{x}\|\}=2\|H\| \cdot\|x\| \\
& =2\|F-G\|\|x\| \leq 2 \delta\|x\|=\varepsilon\|x\|
\end{aligned}
$$

thus proving (6.9) and therefore (6.8). (6.4) follows now from (6.6) and (6.7).

REMark 6.1. Let $R$ be a real Banach space. Let $D$ be a bounded open set of $R, \bar{D}$ its closure, $B$ its boundary, and let (6.1) be a map of $\bar{D}$ into $R$ with completely continuous (not necessarily linear) $F(x)$. We then call $f(x)$ smooth if it satisfies conditions i) and ii) of definition 5.1 and if in addition the linear operator in $h, D(x, h)=h-d(x, h)$ is completely continuous (cf. 
Remark 5.1). Perusal of section 5.1 shows then that everything said in this section remains valid if the following substitutions are made throughout: i) $X$ is replaced by $R$; ii) definition 5.1 of a smooth map is replaced by the definition just given; iii) references to theorem 2.1 are replaced by references to theorem 6.1, and definition 2.1 by definition 6.2 .

By the preceding remark the degree is defined and homotopy properties are established for smooth mappings in a real Banach space $R^{36}$ ).

\section{Bibliography.}

[1] DUNFoRd, N.: Spectral theory. I. Convergence to projections. Trans. Amer. Math. Soc. 54, 185-217 (1943). - [2] Graves, L. M.: Riemann integration and Taylor's theorem in general analysis. Trans. Amer. Math. Soc. 29, 163-177 (1927). - [3] Graves, L. M., and T. H. HILDEBRANDT: Implicit functions and their differentials in general analysis. Trans. Amer. Math. Soc. 29, 126-153 (1927). - [4] HILlE, E.: Functional analysis and semi-groups. Amer. Math. Soc., Colloquium Publications 21 (1948). [5] Leray, J.: La theorie des points fixes et ses applications en analyse. Proc. Internat. Congress of Mathematicians, Cambridge, Mass. U.S.A., 1950. Publ. Amer. Math. Soc. 2, 202-207 (1952). - [6] LERAY, J., et J. SchaUdER: Topology and equations fonctionnelles. Ann. sci. École norm. sup. (3) 51, 45-78 (1934). - [7] Mackey, G. W.: Isomorphisms of normed linear spaces. Ann. Math. 43, 244-260 (1942). - [ [8] NaGUMo, M.: A theory of the degree of mapping based on infinitesimal analysis. Amer. J. Math. 73, 485-496 (1951). - [9] NAGUMO, M.: Degree of mapping in convex linear topological spaces. Amer. J. Math. 73, 485-496 (1951). - [10] ScHWARTz, J.: Perturbations of spectral operators and applications. I. Bounded perturbations. Pacific J. Math. 4, 415-458 (1954). - [11] STONE, M. H.: Linear transformations in Hilbert space and their applications to analysis. Amer. Math. Soc., Colloquium Publications 15 (1932).

${ }^{36}$ ) This degree agrees with the one defined by Leray and Schauder. The latter, however, applies to a wider class of mappings.

Dept. of Math., University of Michigan, Ann Arbor, Mich. (U.S.A.).

(Eingegangen am 16. März 1955.) 